\title{
Food Safety Strategies: The One Health Approach to Global Challenges and China's Actions
}

\author{
Di Wu'; Christopher Elliott ${ }^{1, *}$; Yongning $\mathrm{Wu}^{2, *}$
}

\section{WORLD FOOD SAFETY DAY}

World Food Safety Day (WFSD) (1) celebrated on 7 June 2021 aims to draw attention and inspire action to help prevent, detect and manage foodborne risks, contributing to food security, human health, economic prosperity, agriculture, market access, tourism and sustainable development.

The theme of 2021, "Safe food today for a healthy tomorrow", stresses that production and consumption of safe food has immediate and long-term benefits for people, the planet and the economy. Recognizing the systemic connections between the health of people, animals, plants, the environment and the economy will help us meet the needs of the future.

Recognizing the global burden of foodborne diseases, which affect individuals of all ages, in particular children under-5 and persons living in low-income countries, the United Nations General Assembly proclaimed in 2018 that every 7 June would be World Food Safety Day. In 2020, the World Health Assembly (WHA) further adopted a decision on strengthening efforts on food safety to reduce the burden of foodborne disease. The World Health Organization (WHO) and the Food and Agriculture Organization of the United Nations (FAO) jointly facilitate the observance of World Food Safety Day, in collaboration with Member States and other relevant organizations.

Food safety is a shared responsibility between governments, producers and consumers. Everyone has a role to play from farm to table to ensure the food we consume is safe and healthy. Through the World Food Safety Day, WHO works to mainstream food safety in the public agenda and reduce the burden of foodborne diseases globally. Food safety is everyone's business.

\section{WHY AN UPDATED GLOBAL FOOD SAFETY STRATEGY?}

At the 73rd WHA forum held in May 2020, WHO called for experts to advise the body's work to update its global strategy for food safety (2) and aimed to deliver a new plan by 2022. Thus, to address the most emerging challenges tout de suite and to strengthen and harmonize international food safety systems. The incorporation of innovative technologies and approaches is required to tackle existing and new threats to the safety of the food supply and to public health. As recorded in the Resolution WHA73.5, "Strengthening Efforts on Food Safety" (3), reaffirmed that food safety remains an essential element of public health, with the highest political level priority, and is a means to implement the 2030 Agenda for Sustainable Development. The resolution further recognized the need of collective action throughout all stages of the supply chain at the local, national, regional, and global levels. It also called on Member States to develop food safety policies that take into consideration all stages of the supply chain, the best available scientific evidence, advice, and innovations; to provide adequate resources to improve national food safety systems; to recognize consumer interests; and to integrate food safety into national and regional policies on health, agriculture, trade, environment, and development.

In many jurisdictions, oversight of the food chain is fragmented with different ministries and professionals responsible for different segments and often there are gaps and inconsistencies in the continuum of oversight from farm to fork. The multidisciplinary and multiagency One Health holistic approach must be adopted if existing and emerging problems are to be tackled effectively.

The WHO has outlined five strategic priorities to develop this Global Strategy for Food Safety based on situational assessment and multiple consultations with its Member States, subject matter experts, regional advisers in 
food safety, intergovernmental, non-governmental organizations, private sector, and in addition, the Regional Framework for Action on Food Safety in the WHO Regional Office (Figure 1).

WHO has established the Technical Advisory Group (TAG) on food safety to draft this strategy in order to adds value by providing an overall vision and strategic priorities for concerted global action and by underlining the importance of food safety as a public health priority and the need for enhancing global cooperation across the whole food and feed chain. The strategy also reflects, and is complementary to, existing WHO health programs, such as nutrition and non-communicable diseases, antimicrobial resistance, public health emergency and emerging diseases, climate change, environmental health, water and sanitation, and neglected tropical diseases.

\section{THE AIM AND VISION OF THE FOOD SAFETY STRATEGY}

The Global Food Safety Strategy (4) has been developed to guide and support Member States to prioritize, plan, implement, monitor, and regularly evaluate actions towards the reduction of the incidence of foodborne diseases by continuously strengthening food safety systems and promoting global cooperation.

The vision of the strategy is to provide safe and healthy food for all. All countries are essential stakeholders in food safety to promote, support and protect public health and reduce the burden of foodborne diseases.

\section{FOOD SAFETY: A PUBLIC HEALTH PRIORITY}

Foodborne diseases have enormous impacts on public health. Unsafe food, containing harmful levels of bacteria, viruses, parasites, or chemical or physical substances, contribute to acute or chronic illnesses, with more than 200 consequential diseases and conditions - ranging from diarrhea to cancers to permanent disability or death. An estimated 600 million, almost 1 in 10 people in the world, fall ill after eating contaminated food, resulting in a global annual burden of 33 million disability-adjusted life years (DALYs) and 420,000 premature deaths (5). Unsafe food disproportionately affects vulnerable groups in society, particularly infants, young children, the elderly, and the sick. Low- and middle-income countries are the most affected, with an annual estimated cost of 110 billion USD in productivity losses, trade-related losses, and medical treatment costs due to the consumption of unsafe foods ( 6 ). Moreover, the globalization of the food supply means that populations worldwide are increasingly exposed to new and emerging risks, such as emerging pathogens, existing pathogens with new virulence traits and the development of antimicrobial resistance (AMR) in foodborne pathogens. It was estimated that by 2050, 10 million lives will be at

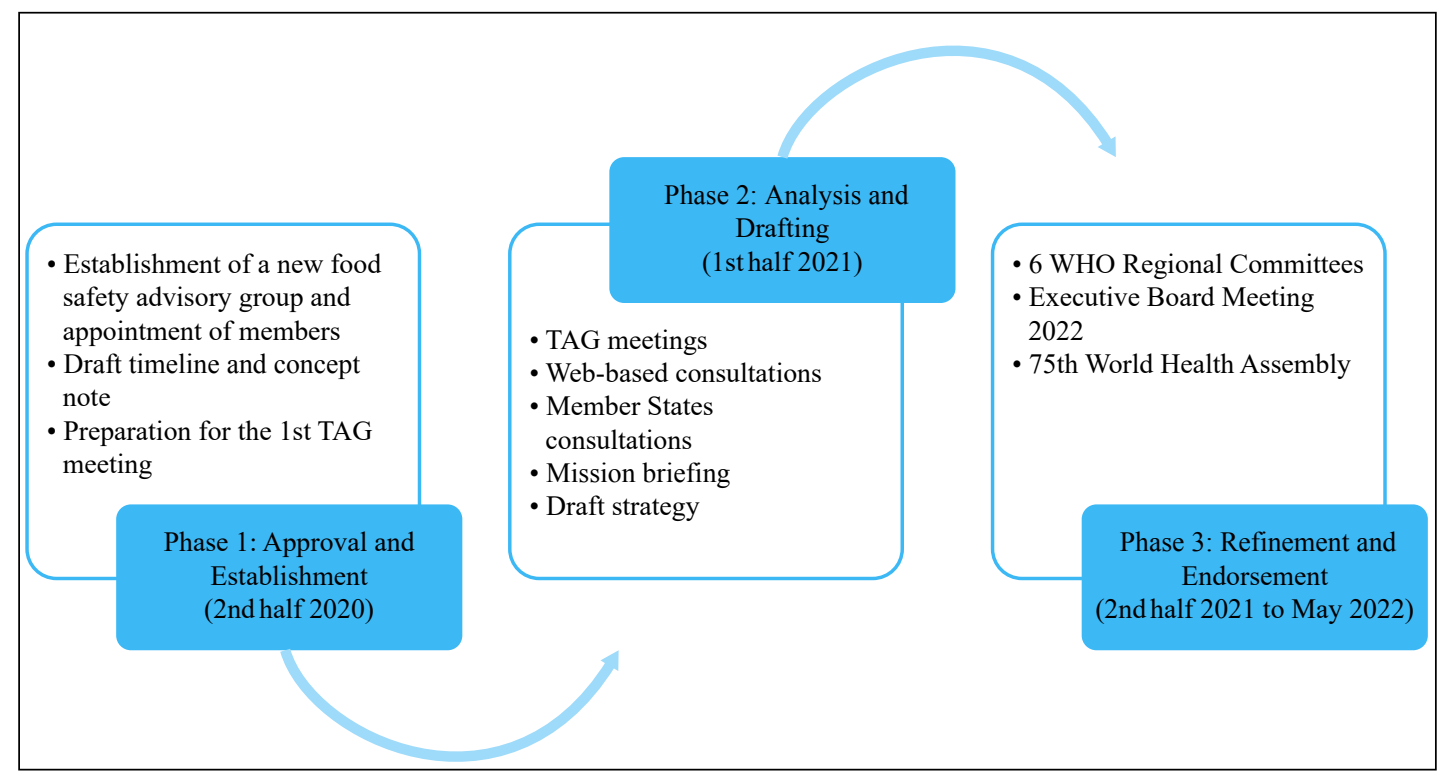

FIGURE 1. The overall process for the update of the WHO Global Strategy for Food Safety.

Abbreviation: TAG=Technical Advisory Group. 
risk and a cumulative 100 trillion USD will be lost due to the spread of AMR if no proactive solutions are taken (7). One of the main sectors of antimicrobial usage is the food system. AMR arises from the inappropriate use of antimicrobials in humans and in food producing animals, so the food production system has a role to tackle the problem.

\section{DRIVERS OF THE CHANGE IN FOOD SAFETY}

Numerous factors impact on food systems and influence the safety of the food supply. While it is not always possible for agencies of government with responsibility for food safety to control these "drivers," it is important to recognize and understand their influence on existing and emerging food safety risks. In strengthening any national food control system, the key drivers Member States need to be aware of are summarized as the following: 1) Stakeholder interests and demands for safe food; 2) Global food safety threats; 3) Global changes in the economics of the food supply, 4) Environmental and climate change challenges; 5) Shifts in consumer preferences and expectations; 6) Rise of new technologies and digital transformation; 7) Population demographics.

\section{FOOD SAFETY DEMANDS A ONE HEALTH APPROACH}

It is now widely recognized that the health of people is closely connected to the health of animals and our shared environment (Figure 2) (8). With rapid population growth, globalization, and environmental degradation, threats to public health have become more complex. Recent emerging diseases such as Middle East respiratory syndrome (MERS), Ebola, and H7N9, have all been linked to our food systems and the environment. The COVID-19 pandemic has shown how vulnerable the global population is to the undetected emergence of new diseases, particularly zoonoses that originate at the human-animal-environment interface. Food production, intensive agriculture, livestock systems, wildlife trade and humans encroaching on wildlife habitats and weather-related disasters all contribute to increasing the risk of emergence of new zoonotic diseases. Mitigation of these threats cannot be achieved by one sector acting alone.

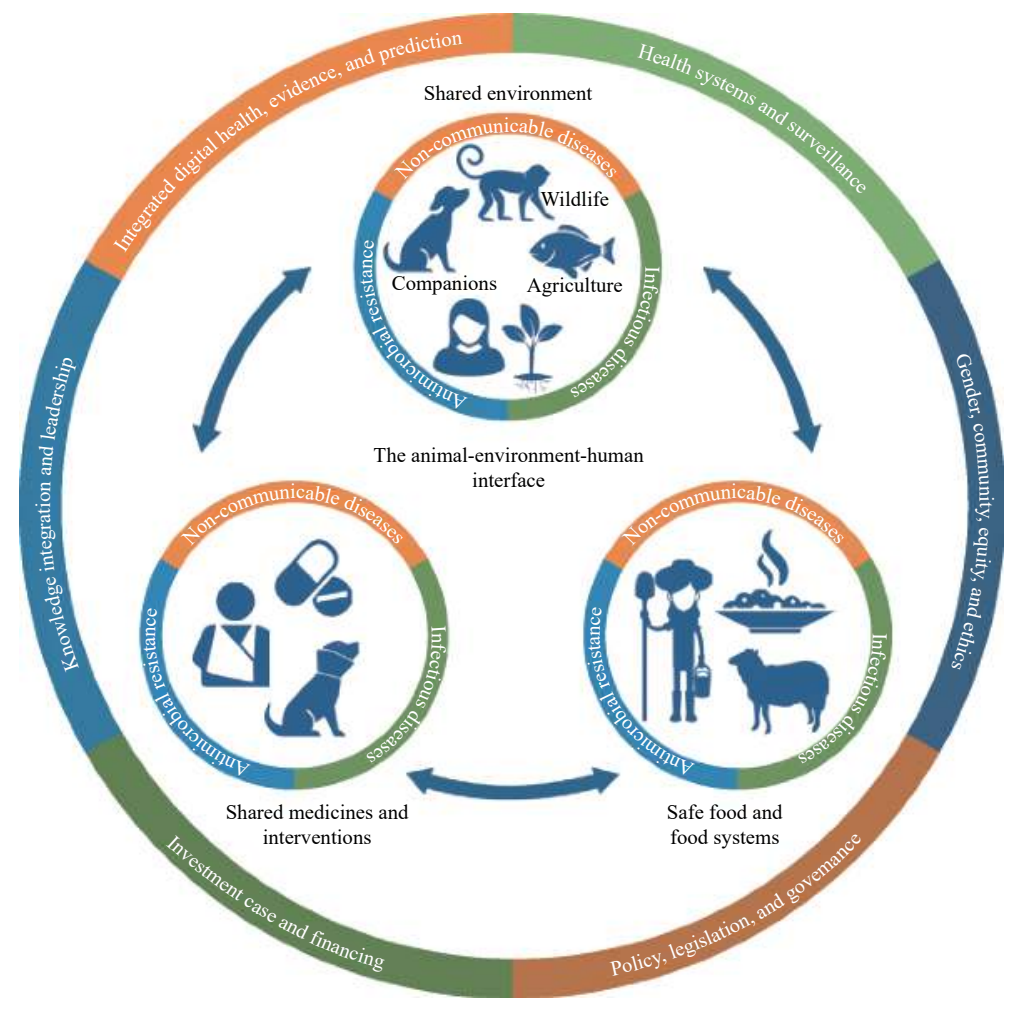

FIGURE 2. One Health approach: tackling health risks at animal-environment-human interface. 
The One Health approach must be adopted if emerging diseases are to be detected and controlled at source. Whole genome sequencing is providing the ability to compare isolates from the environment, livestock, food and humans and track pathogens to where corrective actions are required. Future improvements in food safety and public health will largely depend on how well sectors manage to collaborate using the One Health approach. Data on the occurrence of and disease burden from foodborne hazards combined with knowledge of source attribution will be crucial in assessing costs and benefits of novel control measures. One Health collaboration will enable the necessary integration of data to inform preventive actions at the appropriate stages of the food chain. Without knowledge of the incidence and burden of disease, and the source of contamination, associated with hazard/food combinations, prioritization of mitigation action will be difficult and food safety improvements will be largely unsuccessful.

Many food related to chemical hazards, both from natural and manmade sources, reach consumers from or via animals or the environment and should be covered within the One Health framework. Chemical food contamination is a major cross-cutting issue with many agrochemicals and antimicrobials used in plant and animal production. In addition, the naturally occurring toxins, such as mycotoxins, present an ongoing challenge and an increasing threat due to climate change. Therefore, One Health monitoring and surveillance systems should clearly include natural and manmade chemical hazards.

Climate change is a major and growing influencing factor of food systems and is likely to have considerable negative impacts on food security, nutrition, and food safety. By modifying the persistence and transmission patterns of foodborne pathogens and contaminants, climate change leads to the escalation of foodborne risks (9). In this regard, food safety should also be integrated into interventions and commitments for climate change adaptation and mitigation under a One Health approach.

Adopting a One Health approach to food safety will allow Member States to detect, prevent and respond to emerging diseases at the human-animal-environment interface and to address food-related public health issues more effectively.

\section{SCOPE OF THE STRATEGY}

Strengthening national food safety systems begins with establishing or improving infrastructure and components of food control systems as described in Strategic Priority 1. For example, these may include developing framework food legislation, standards and guidelines, laboratory capacity, food control activities and programs, and emergency preparedness capacity. In addition to establishing a national food control system, four important characteristics/principles (Figure 3) (10) need to be considered and adopted for the system to be fully operational. The five strategic priorities are based on the fundamental components/infrastructure of the food safety systems and four additional principles.

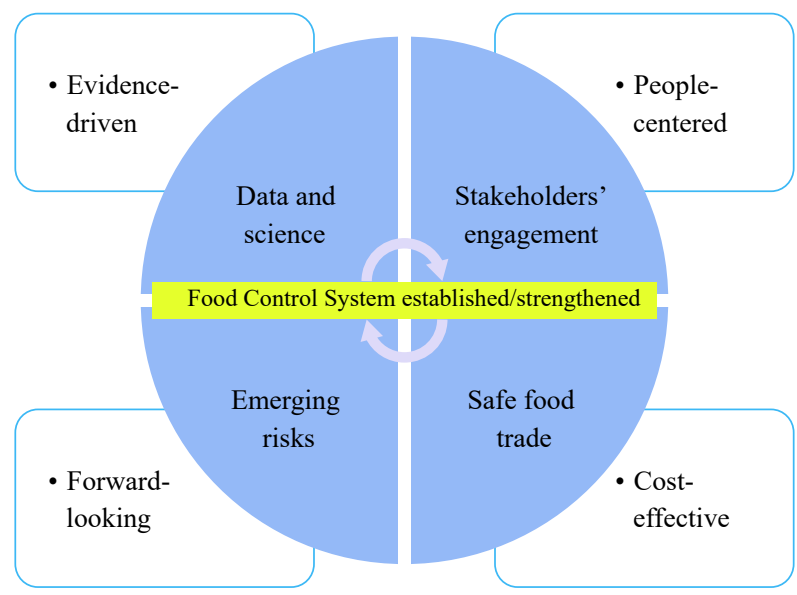

FIGURE 3. Conceptual framework for strategic priorities. 


\section{FIVE STRATEGIC PRIORITIES}

It is intended that the new global strategy will provide an overall vision and strategic priorities for concerted global action that will underline both the importance of food safety as a public health priority and the need to enhance its critical role as a public health component in food systems. In discussing the strategic priorities, some participants suggested that the broad focus should be on national food safety systems rather than on national food control systems. Food systems encompass the entire range of actors and their interlinked value-adding activities involved in the production, aggregation, processing, distribution, consumption, and disposal of food products that originate from agriculture, forestry or fisheries, and parts of the broader economic, societal, and natural environments in which they are embedded. In the context of a food systems approach, the national food safety system would be the combination of activities of all stakeholders in the food chain to safeguard the health and wellbeing of people, while fostering economic development and improving livelihoods by promoting access to domestic, regional, and international markets.

The different components of the national food safety system would include, but would not be limited to, the national food control system (official food controls conducted by government agencies); food safety management systems (risk-based systems based on Hazard Analysis and Critical Control Point (HACCP) principles conducted by food business); foodborne disease surveillance systems (responsibility of the health sector); national food monitoring system for pesticide/residues/mycotoxin contaminants (part of official controls conducted by government agencies); animal disease surveillance systems (part of official controls conducted by veterinary agencies). Animal disease surveillance is important both for zoonotic pathogens and for animal specific disease. Many animal specific diseases can disrupt supply changes, e.g. Foot and Mouth Disease and African Swine fever and sick animals require antimicrobial treatment which can trigger the development of AMR in non-target microbes.

For the purposes of this strategy and to ensure alignment with the standards, guidelines, and codes of practice of the Codex Alimentarius, the WHO, in consultation with the FAO, has proposed that the term national food control system will be used where referring to the national food safety system. A national food control system of policies, procedures, and plans, includes a mandatory regulatory approach together with scientific information and preventative educational strategies that protect the whole food chain. This includes effective enforcement of food legislation, along with training and education, community outreach programs, and promotion. TAG members noted the importance of aligning with the Codex Alimentarius on the usage of terminology. However, concerns were also expressed as "food safety systems" is the terminology used in the WHA73.5 and the usage of "food control systems" can create an impression with those who are not familiar with the Codex standards, that the strategy only focuses on the control functions carried out by governments while excluding the activities of other relevant stakeholders in food safety. The 5 Strategic Priorities agreed by participants are:

- Strategic Priority 1: Strengthening national food control systems.

- Strategic Priority 2: Identifying and responding to food safety challenges resulting from the transformation and global changes in food systems.

- Strategic Priority 3: Increasing the use of food chain information, scientific evidence, and risk assessment in making risk management decisions.

- Strategic Priority 4: Strengthening stakeholder engagement and risk communication.

- Strategic Priority 5: Promoting food safety as an essential component in domestic and international trade.

An additional strategic priority was proposed to include technical cooperation to enhance the food safety situation in developing countries. Fostering regional and global cooperation and international connectivity should be a key theme for the strategy.

Countries have flexibility to determine how best to design their food control system and implement a wide range of control measures. The Codex Alimentarius Principles and Guidelines for National Food Control Systems will assist Member States in reviewing and strengthening their national systems (11). While recognizing the diversity of national food control systems at different levels of development and the wide range of food safety hazards, FAO and WHO have developed a framework for developing national food safety emergency response plans to assist Member States to develop country-specific plans (12). Today's global challenges are transforming the way we produce, market, consume, and think about food (13). The provision of a long-term safe, nutritious, and affordable food 
supply is a global endeavor and how we grow, produce, and sell food impacts us all, either as stakeholders in national and global agri-food value chains or as consumers of the increasing variety of food that is produced domestically or imported. The complexity of global food systems, and the speed at which they can change, demands that governments and competent authorities have a clear view of the connectedness between the global and regional food systems within which food is produced, distributed, and sold, and the food control system they regulate. Food safety is a core enabling factor to successfully transform food systems and Member States need to be aware of food safety issues as the transformation of food systems accelerates.

In many countries, different government ministries have a strong interest in decisions on food control measures made by the competent authority and their inputs may need to be considered as part of the decision-making process. Competent authorities can benefit from the use of international guidelines on multi-factor decision-making to promote consistency and transparency in their choice of control measures (14). A One Health approach to risk management generally involves cross-disciplinary inputs when responding to new or emerging risks arising at the human-animal-plant-environmental interfaces. As health threats become more complex, mitigation cannot be achieved by one sector acting alone. Food safety authorities may have to factor in public, veterinary, and environmental health considerations in establishing control measures. As an example, use of antimicrobials of critical importance (15) to public health may require their partial, or even, total withdrawal from use in food animal production because of the likelihood of antimicrobial resistance.

\section{CHINA'S NATIONAL FOOD SAFETY STRATEGY AND ITS STRATEGIC PRIORITIES}

The national food safety strategy proposed by China marks the foundation of a unique Chinese framework in food safety managing system with a core goal to ensure its people "eat at ease and safely." An entire food chain approach from animal feed production right through to consumption by the final consumer will be adopted. Consistent oversight of the food chain with equal risks receiving equal amounts of attention will be adopted. There will not be degrees of safety and food for the domestic market and for export will meet the same food safety standards.

Under the state-level guiding principle of "integrated marketing, supervision, industry, and management," the strategy focuses on harmonizing the domestic food market, optimizing government supervision, promoting highquality development, and coordinated social governance. In addition, this strategy is supported with increased financial investment, education, and related regulations. Specific measures include: 1) establishing a unified, modern, open market system with managed competition; 2) promoting optimization, collaboration, and efficiency of the supervision system; and 3) establishing a social governance model based on collaboration, participation, and common interests. Therefore, as a concrete application of the WHO strategy, the Chinese government has proposed its own timetable and roadmap for its domestic food safety strategy: 1) zero tolerance of systemic food safety risks by 2020 and constantly improving the level of food safety assurance; 2) establishing a strict, highly efficient, and socially-governed food safety governance system by 2027;3) achieving the modernization of food safety governance by 2035; and 4) achieving universal modernization of food safety governance and approaching world's top food safety level ranking by 2050 .

Acknowledgement: The Horizon 2020 EU-China-Safe program that was jointly founded by EU-China Flagship Project on Intergovernmental Cooperation of S\&T Innovation from the Chinese Ministry of Science and Technology (grant No. 2017YFE0110800) and the European Commission (H2020 grant No. 727846); NSFC project 31801454 and Newton International Fellowship NIF|R1\192293 of Royal Society.

doi: $10.46234 / \mathrm{ccdcw} 2021.131$

\# Corresponding authors: Christopher Elliott, Chris.elliott@qub.ac.uk; Yongning Wu, wuyongning@cfsa.net.cn.

\footnotetext{
${ }^{1}$ Institute for Global Food Security, Queen's University Belfast, Belfast, the United Kingdom; ${ }^{2}$ National Health Commission Key Laboratory of Food Safety Risk Assessment, Food Safety Research Unit (2019RU014) of Chinese Academy of Medical Science, China National Center for Food Safety Risk Assessment, Beijing, China.
} 


\section{REFERENCES}

1. Food and Agriculture Organization of the United Nations and World Health Organization. Observances: Food safety day. https://www.un. org/en/observances/food-safety-day. [2021-6-9].

2. WHO Food Safety Programme. WHO global strategy for food safety: safer food for better health. https://apps.who.int/iris/handle/10665/42559. [2021-6-9].

3. The 73rd World Health Assembly. Strengthening efforts on food safety. 2020. https://apps.who.int/gb/ebwha/pdf_files/WHA73/A73_R5-en.pdf. [2021-69].

4. World Health Organization. Public consultation on the draft WHO global strategy for food safety. Geneva: World Health Organization, 2021. https://www.who.int/news-room/articles-detail/public-consultation-on-the-draft-who-global-strategy-for-food-safety.

5. WHO Foodborne Disease Burden Epidemiology Reference Group. WHO Estimates of the global burden of foodborne diseases. (2007-2015). Geneva: World Health Organization, 2015. https://apps.who.int/iris/bitstream/handle/10665/199350/9789241565165_eng.pdf?sequence=1.

6. Steven J, Spencer H, Laurian U, Delia G, Emilie C. The safe food imperative: accelerating progress in low- and middle-income countries. Agriculture and Food Series. Washington, DC: World Bank, 2019. https://openknowledge.worldbank.org/handle/10986/30568.

7. O'Neill J. Tackling drug-resistant infections globally: final report and recommendations. 2016. https://www.scirp.org/reference/referencespapers. aspx?referenceid $=2510715$. [2021-6-9].

8. Amuasi JH, Lucas T, Horton R, Winkler AS. Reconnecting for our future: The Lancet one health commission. Lancet 2020;395(10235):1469 - 71. http://dx.doi.org/10.1016/S0140-6736(20)31027-8.

9. World Health Organization. Food safety-climate change and the role of WHO. Geneva: World Health Organization, 2019. https://www.who.int/ foodsafety/publications/all/climate_change/en/.

10. World Health Organization. Draft WHO global strategy for food safety 2022-2030. https://cdn.who.int/media/docs/default-source/food-safety/publicconsultation/draft-who-global-strategy-for-food-safety-13may2021.pdf?sfvrsn=ac480bb9_5. [2021-6-9].

11. Food and Agriculture Organization of the United Nations. Principles and guidelines for national food control systems. CAC/GL 82-2013, 2013. https://www.fao.org/fao-who-codexalimentarius/sh-proxy/en/?lnk=1\&urlhttps\%253A\%252F\%252Fworkspace.fao.org\%252Fsites\%252Fcodex \%252 FStandards\%252FCXG\%2B82-2013\%252FCXG_082e.pdf. [2021-6-9].

12. Food and Agriculture Organization of the United Nations and World Health Organization. FAO/WHO framework for developing national food safety emergency response plans. Rome: Food and Agriculture Organization of the United Nations and World Health Organization, 2010. http://www. fao.org/3/i1686e/i1686e00.pdf.

13. Food and Agriculture Organization of the United Nations. The future of food safety. http://www.fao.org/3/CA3247EN/ca3247en.pdf. [2021-6-9].

14. Food and Agriculture Organization of the United Nations. Food safety risk management: evidence-informed policies and decisions, considering multiple factors. http://www.fao.org/documents/card/en/c/I8240EN/. [2021-6-9].

15. World Health Organization. WHO list of critically important antimicrobials for human medicine (WHO CIA list). Geneva: World Health Organization. https://apps.who.int/iris/handle/10665/325036.

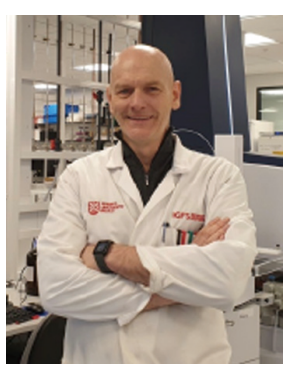

\section{Christopher Elliott, PhD}

Professor of Food Safety;

Director of the ASSET (Assured, Safe and Traceable) Technology Centre;

Pro Vice Chancellor (2015-2018);

Founder Director of Institute for Global Food Security;

Queen's University Belfast, Belfast, the United Kingdom.

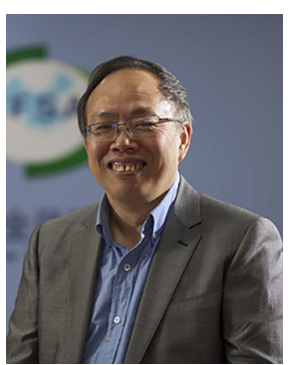

Yongning $\mathrm{Wu}, \mathrm{MD}, \mathrm{PhD}$

Chief Scientists, China National Center for Food Safety Risk Assessment;

Director of NHC Key Laboratory of Food Safety Risk Assessment;

Director of Chinese Academy of Medical Science Research Unit (2019RU014);

Member of WHO Technical Advisory Group of Food Safety. 